\title{
The spatial analysis of annual measles incidence and transition threat assessment in Iran in 2016
}

\author{
Abolfazl Mohammadbeigi ${ }^{1}$, Seyed Mohsen Zahraei ${ }^{2}$, Azam Sabouri $^{2}$, Azadeh Asgarian $^{3}$, \\ Sima Afrashteh ${ }^{4}$, Hossein Ansari*5
}

\begin{abstract}
Background: During the past years, due to the increase in immunization coverage and promotion of surveillance data, the incidence of measles decreased. This study aimed to determine the measles incidence risk, to conduct spatial mapping of annual measles incidence, and to assess the transition threat in different districts of Iran.

Methods: A historical cohort study with retrospective data was conducted. The measles surveillance data containing 14294 cases suspected of having measles in Iran were analyzed during 2014-2016. WHO Measles Programmatic Risk Assessment Tool was applied to calculate and map the incidence of measles in each district, to determine the annual incidence rate, and to conduct spatial threat assessment risk. Threat assessment was measured based on factors influencing the exposure and transmission of measles virus in the population. The annual measles incidence rate and spatial mapping of incidence in each Iranian district was conducted by Measles Programmatic Risk Assessment Tool. Data were analyzed by descriptive statistics in Excel 2013 and Arc GIS 10.3.

Results: Of 14294 suspected cases, $0.6 \%$ (CI 95\%: 0.599-0.619) were identified as clinically compatible measles, 0.280 (CI 95\%: $0.275-0.284$ ) as confirmed rubella, $0.52 \%$ (CI 95\%: 0.516-0.533) as epi-linked measles, $4.6 \%$ (CI 95\%: 0.450-0.464) as lab-confirmed measles, and 94\% (CI 95\%: 93.93-94.11) were discarded. The annual incidence rate in cases per 100000 populations increased from 0.0726, (CI 95\%; 0.0714-0.0738) in 2014 to 0.1154 (CI 95\%; 0.1135-0.1173) in 2016, and the 3-year incidence rate during the study period was 1.032 (CI 95\%; 1.017-1.047) and the annual average was 0.3442 (CI 95\%; 0.3387-0.3496).

Conclusion: The average annual incidence rate of measles in Iran was low, but after a 11-fold increase in the number of measles cases in 2015, the number of measles cases decreased to more than 7-times in 2016. However, maintaning a high immunization coverage of measles and timely vaccination can be effective in reaching the goal of measles elimination.
\end{abstract}

Keywords: Incidence rate, Iran, Measles, Surveillance, Spatial mapping

Conflicts of Interest: None declared

Funding: None

*This work has been published under CC BY-NC-SA 1.0 license.

Copyright $\odot$ Iran University of Medical Sciences

Cite this article as: Mohammadbeigi A, Zahraei SM, Sabouri A, Asgarian A, Afrashteh S, Ansari H. The spatial analysis of annual measles incidence and transition threat assessment in Iran in 2016. Med J Islam Repub Iran. 2019 (4 Dec);33:130. https://doi.org/10.47176/mjiri.33.130

\section{Introduction}

According to the World Health Organization (WHO) reports, the measles cases showed a high speed decreasing trend in morbidity and mortality in the recent years (1). Iran is one of the countries in the Eastern Mediterranean Region

\footnotetext{
Corresponding author: Dr Hossein Ansari, ansarih@razi.tums.ac.ir

1. Neuroscience Research Center, Faculty of Health, Qom University of Medical Sciences, Qom, Iran

2. Centre for Communicable Diseases Control, Ministry of Health and Medical Education, Tehran, Iran

3. Qom University of Medical Sciences, Qom, Iran

4. Bushehr University of Medical Sciences, Bushehr, Iran

5. Health Promotion Research Center, Department of Epidemiology and Biostatistics, Zahedan University of Medical Sciences, Zahedan, Iran
}

(2). During the past years, the routine immunization coverage was increased and the surveillance system data were improved and, consequently, the disease incidence decreased (3-7). Thus, the endemic virus transmission have

$\uparrow$ What is "already known" in this topic:

During the past years, the routine immunization coverage of measles was increased and the surveillance quality was improved. Consequently, the incidence of measles decreased and the endemic virus transmission was interrupted in Iran.

\section{$\rightarrow$ What this article adds:}

The annual incidence rate of measles in Iran was low; the average annual incidence rate was 3.442 per 1 million people. The annaul incidence rate in 2014 was 0.726 per 1 million, which suddenly increased to more than 11-fold in 2015 . However, the annual incidence rate decreased to 1.154 per 1 million in 2016 by supplimentary immunization activities. 
already been interrupted in Iran (2).

In the early 1980s, regular outbreaks of measles occurred in Iran and 30000 to 50000 cases of measles were reported annually (2). After starting the expanded immunization program in Iran, the number of measles cases decreased and by the nationwide campaign of measles-rubella in 2003, the measles cases decreased to zero (2-4). During the 5 years after the catch-up campaign during 2004-2008, the incidence of measles was 0.2 cases per 1000000 persons and 103 laboratory-confirmed measles cases were registered (8). According to our recent study, the overall immunization coverage for all routine vaccines in Iran was $96.8 \%$ (5). Moreover, the coverage of MMR1 and MMR2 vaccine in the outskirt area of large Iranian cities was estimated as $97.1 \%$ and $94.9 \%$, respectively $(5,9)$. However, the highest delay in vaccination occurred in MMR2 with $74.9 \%$ (9). However, from 2000, effective efforts to eradicate measles have slowed, as the disease and mortality rates decreased in the world $(2,7)$. Nevertheless, based on our recent analysis, there was not any inequity in immunization coverage among Iranian children according to demographic characteristics; however, the prevalence of immunization delay was higher in migrant population (10). Moreover, inadequacy in MMR immunization is related to place of living, mother's education, and immigration, which can be associated with occurrence of measles (11). This study aimed to determine measles incidence risk, to conduct spatial mapping of annual measles incidence, and to assess the transition threat in different districts of Iran.

\section{Methods}

The measles surveillance data of Iran during 2014-2016 were analyzed in a historical cohort study with retrospective data. The measles surveillance system in Iran, as a robust communicable diseases surveillance system, is responsible for collecting and reporting surveillance data (12) and, based on WHO standard recommendations, should detect and report all measles cases (2). Moreover, measles surveillance data, the mapping of all districts of Iran in the Geographic Information System (GIS) shape file, population size in 2016 census for each district, and the EPI manager assessment at the national or district level were used in data analysis. Based on the population size in 2016 census in Iran and the area of each district, population density was estimated and the annual incidence rate for measles was calculated. Finally, according to the incidence rate in 2016, the spatial mapping of measles incidence was depicted for each district. Moreover, the population size for Iran in 2014 and 2015 was estimated by growth rate $(1.29 \%)$ and the population size in 2011 census using direct method estimation (13). The total population of each year was considered as the denominator for the estimation of the incidences.

The measles surveillance data included the year, province, reporting district, case ID, and final classification of measles based on data file including lab-confirmed measles, epi-linked measles, clinically compatible measles, confirmed rubella, discarded and pending), and other data in the surveillance data sheet as described by WHO guideline (14). During the 3-year study period, 14294 suspected measles cases were assessed in Iran. The classification of disease and the vaccination status of suspected cases were assessed. Four measles classification in surveillance data were used in data analysis. The clinical case of measles is defined as a person with fever and maculopapular rash that have cough, coryza, or conjunctivitis and have the measlesspecific IgM antibodies. However, the laboratory classification outline should be applied in countries with low incidence or elimination phase. The laboratory classification scheme to define each case classification, including laboratory-confirmed, epidemiologically confirmed, clinically confirmed, and discarded, was based on WHO protocol of measles surveillance (15). During 2014-2016, the number of lab-confirmed measles cases was 653, epi-linked measles 75 , and clinically compatible measles 87 , respectively. Therefore, the total number of measles cases during 2014 to 2016 was 815 .

Threat assessment of measles is evaluated based on factors that may influence the risk of exposure and transmission of measles virus among people. The threat assessment indicators are based on 4 indexes: (1) reported measles cases among specific age groups, (2) recent measles cases reported in a bordering district, (3) population density, and (4) presence of vulnerable population groups (VPGs). The presence of VPG was assessed by local knowledge of the EPI manager at the national or district level. These groups are defined by WHO and based on the guideline of measles programmatic risk assessment tool (14). Moreover, these definitions for VPG were used in other studies $(1,16,17)$. VPGs included 8 different factors in people that increased the risk of measles outbreaks, which have been mentioned in our recent study (7).

This study was approved by the ethical committee of Qom University of Medical Sciences (IR.MUQ.REC. 197.029 code). The authors reported the overall results of the study, and thus it was impossible to identify the patients.

\section{Data analysis}

The WHO measles programmatic risk assessment tool was used for data analysis. This tool was developed to estimate the risk point score of measles outbreak in each region based on surveillance data, population size, and vaccination coverage, surveillance quality indicators of measles, and presence of VPGs. Moreover, the shape files of all Iranian districts were used to map the spatial incidence of measles. Furthermore, the annual measles incidence rate and spatial mapping of incidence in each Iranian district was conducted by this online software $(14,18)$. More detail about the measles programmatic risk assessment tool and calculation of risk score is provided in our recent studies $(7,19)$.

\section{Results}

During the study years, 14294 suspected cases were assessed, and the final classification of the suspected case showed that $0.6 \%$ of the cases (CI 95\%: $0.599-0.619$ ) $(\mathrm{n}=87)$ were clinically compatible measles, 0.3 (CI 95\%: $0.275-0.284)(\mathrm{n}=40)$ confirmed rubella, $0.52 \%$ (CI 95\%: $0.516-0.533)(\mathrm{n}=75)$ epi-linked measles, $4.6 \%$, (CI 95\%: $0.450-0.464,(\mathrm{n}=653)$ lab-confirmed measles, and $94 \%(\mathrm{CI}$ 95\%: 93.93-94.11) $(\mathrm{n}=13$ 439) were discarded. 
The mean age of the suspected cases was $5 \pm 9.3$ years and varied between 0 to 86 years. However, the mean age in confirmed measles cases was $9.07 \pm 13.95$ years old. In addition, the mean age of confirmed measles cases decreased from 9.45 years to 6.90 years from 2014 to 2016 and the median age of cases was higher in 2015 (2.95 years old) than in 2014 (2.4) and 2016 (1.57). During the 3-year study period, of all cases, $47.2 \%$ (383 cases) were female and $52.8 \%$ (429 cases) were male, and the sex ratio was 1.12 . During the study period, between 2014 to 2016 , the incidence of measles in all age groups was decreased, but this decrease was higher in 2016 than in 2014. Of the cases reported in 2014, more than 50\% were younger than 5 years old. Based on the results, the percentage of cases who were vaccinated increased from $25.9 \%$ in $2014,29.2 \%$ in 2015 , and $51.1 \%$ in 2016 (Table 1 ).

The annual incidence rate of measles per $100000 \mathrm{popu}-$ lations increased from 0.0726 , (CI 95\%; 0.0714-0.0738) in 2014 to 0.1154 (CI 95\%; 0.1135-0.1173) in 2016; and the 3 -year incidence rate was 1.032 (CI 95\%; 1.017-1.047) and the annual average was 0.3442 (CI 95\%; 0.3387-0.3496).

Figure 1 shows that of 322 studied districts of Iran, 33 districts (SavojBolaq in Alborz, Bile Savar in Ardabil, Hashtrud in Esat Azarbayjan, Abadeh, Larestan, Sepidan, Shiraz in Fars, Rasht in Gilan, Bandar Abas, Jask, Minab in Hormozgan, Tiran o Karvan in Isfahan, Bam and Sirjan in Kerman, Kangavar in Kermanshah, Torbat e Jam in Khorasan.Razavi, Bandar Mahshahr, Dezful, Hendijan, Omidiye

Table 1. The characteristics of Measles cases based on Measles classification, vaccine status, age group, gender

\begin{tabular}{|c|c|c|c|c|c|}
\hline Characteristics of Measles cases & Subgroups & & $\begin{array}{c}\text { Year } \\
\text { n }(\%)\end{array}$ & & Total \\
\hline \multirow{4}{*}{ Measles classification } & & 2014 & 2015 & 2016 & \\
\hline & Lab-Confirmed & $40(69)$ & $552(82.8)$ & $61(67.8)$ & $653(80.1)$ \\
\hline & Clinically Compatible & $17(29.3)$ & $54(8.1)$ & $16(17.8)$ & $87(10.7)$ \\
\hline & EPI-Linked & $1(1.7)$ & $61(9.1)$ & $13(14.4)$ & $75(9.2)$ \\
\hline \multirow[t]{3}{*}{ Vaccine Status } & Vaccine & $15(25.9)$ & $195(29.2)$ & $46(51.1)$ & $256(31.4)$ \\
\hline & Not vaccine & $17(29.3)$ & $164(24.6)$ & $6(6.7)$ & $187(22.9)$ \\
\hline & Unknown & $26(44.8)$ & $308(46.2)$ & $38(42.2)$ & $372(45.6)$ \\
\hline \multirow[t]{5}{*}{ Age group } & Lowest 1 year old & $18(31)$ & $250(37.5)$ & $34(37.8)$ & $302(37.1)$ \\
\hline & 1 to 5 years old & $18(31)$ & $124(18.6)$ & $17(18.9)$ & $159(19.5)$ \\
\hline & 5 to 10 years old & $7(12.1)$ & $95(14.2)$ & $13(14.4)$ & $115(14.1)$ \\
\hline & 10 to 20 years old & $3(5.2)$ & $95(14.2)$ & $20(22.2)$ & $118(14.5)$ \\
\hline & Highest 20 years old & $12(20.7)$ & $103(15.4)$ & $6(6.7)$ & $121(14.8)$ \\
\hline \multirow[t]{2}{*}{ Gender } & Female & $34(58.6)$ & $313(47.1)$ & $36(40)$ & $383(47.2)$ \\
\hline & Male & $24(41.4)$ & $351(52.9)$ & $54(60)$ & $429(52.8)$ \\
\hline Total & Measles cases & $58(100)$ & $667(100)$ & $90(100)$ & $815(100)$ \\
\hline Population & & $79926270 * *$ & $78971070 * *$ & $78015780 *$ & 78971070 \\
\hline Incidence rate $(100,000)$ & & 0.0726 & 0.8446 & 0.1154 & 0.3442 \\
\hline
\end{tabular}

*Based on 2016 census

**Based on estimation

\section{Measles Cases In the Past Year}

Any confirmed cases

No confirmed cases

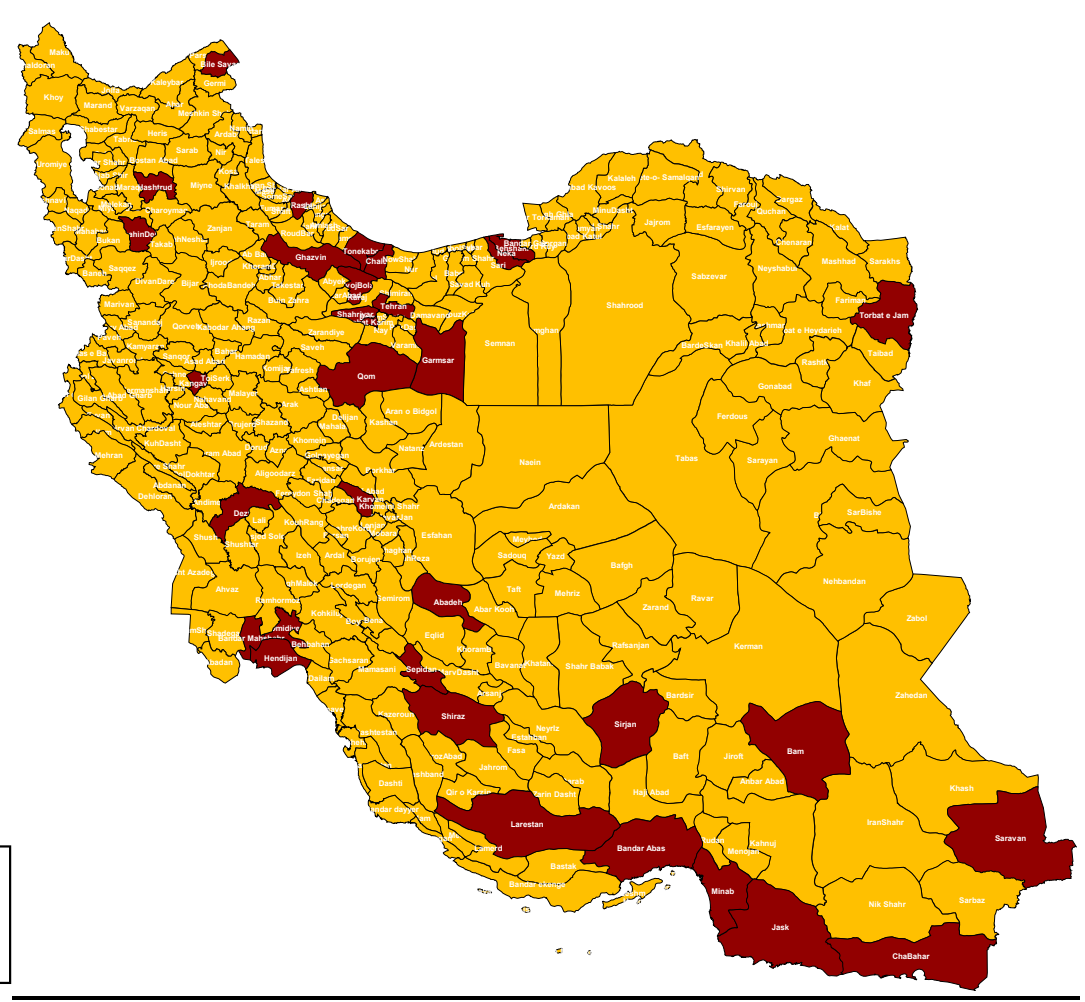

Fig. 1. The mapping of confirmation of any measles cases in 2016 in all Iranian districts 
in Khuzestan, Behshahr, Chalus, Neka and Tonekabon in Mazandaran, Qazvin in Qazvin, Qom, Garmsar in Semnan, ChaBahar and Saravan in Sistan, Robat Karim, Shahriyar and Tehran in Tehran and ShahinDej in West Azarbayjan) had more than 1 confirmed measles case in 2016.

Table 2 demonstrates the annual measles incidence rate per 1000000 in Iranian districts with confirmed measles in 2016. Based on these results, Bandar Mahshahr (92.9), Sepidan (33) and Hedijan (25.8) had the highest annual confirmed measles incidence per 1 million population in Iran. The spatial mapping of annual confirmed measles incidence is depicted in Figure 2.
According to Figure 2, the annual confirmed measles incidence in Bandar Mahshahr was 50-499 per 1000000 population in 2016. The annual confirmed measles incidence in Bile Savar, Hashtrud, Abadeh, Larestan, Sepidan, Bandar Abas, Jask, Minab, Tiran o Karvan, Kangavar, Torbat e Jam, Hendijan, Omidiye, Chalus, Neka, Tonekabon, Garmsar, ChaBahar, Saravan and ShahinDej was between 5-49 per 1000000 population. The annual confirmed measles incidence in SavojBolaq, Shiraz, Rasht, Sirjan, Dezful, Behshahr, Qazvin, Qom, Robat Karim, Shahriyar and Tehran was between 1-4 per 1000000 population.

The results of threat assessment for measles outbreak showed that all Iranian districts were depicted as low risk

Table 2. The annual confirmed measles incidence per 1,000,000 in Iran at 2016

\begin{tabular}{|c|c|c|c|c|c|}
\hline AREA & Annual Incidence & AREA & Annual Incidence & AREA & Annual Incidence \\
\hline Alborz & & Kerman & & Qazvin & \\
\hline SavojBolaq & 3.7 & Bam & 4.6 & Ghazvin & 1.0 \\
\hline Ardabil & & Sirjan & 3.1 & Qom & \\
\hline Bile Savar & 19.5 & Kermanshah & & Qom & 1.5 \\
\hline Esat.Azarbayjan & & Kangavar & 13.3 & Semnan & \\
\hline Hashtrud & 17.5 & Khorasan.Razavi & & Garmsar & 11.0 \\
\hline Fars & & Torbat e Jam & 7.5 & Sistan & \\
\hline Abadeh & 9.9 & Khuzestan & & ChaBahar & 7.9 \\
\hline Larestan & 9.7 & Bandar Mahshahr & 92.9 & Saravan & 8.6 \\
\hline Sepidan & 33.0 & Dezful & 2.3 & Tehran & \\
\hline Shiraz & 0.5 & Hendijan & 25.8 & Robat Karim & 3.4 \\
\hline Gilan & & Omidiye & 17.6 & Shahriyar & 0.7 \\
\hline Rasht & 1.0 & Mazandaran & & Tehran & 0.2 \\
\hline Hormozgan & & Behshahr & 4.8 & West.Azarbayjan & \\
\hline Bandar Abas & 11.8 & Chalus & 7.1 & ShahinDej & 10.8 \\
\hline Jask & 10.6 & Neka & 16.7 & -- & -- \\
\hline Minab & 11.6 & Tonekabon & 9.1 & -- & -- \\
\hline \multicolumn{6}{|l|}{ Isfahan } \\
\hline Tiran o Karvan & 14.0 & -- & -- & -- & -- \\
\hline
\end{tabular}

\begin{tabular}{|l|l|}
\hline Measles Incidence per $1,000,000$ for 2016 \\
0 \\
$\square-4$ \\
$5-49$ \\
$50-499$ \\
500 or more
\end{tabular}

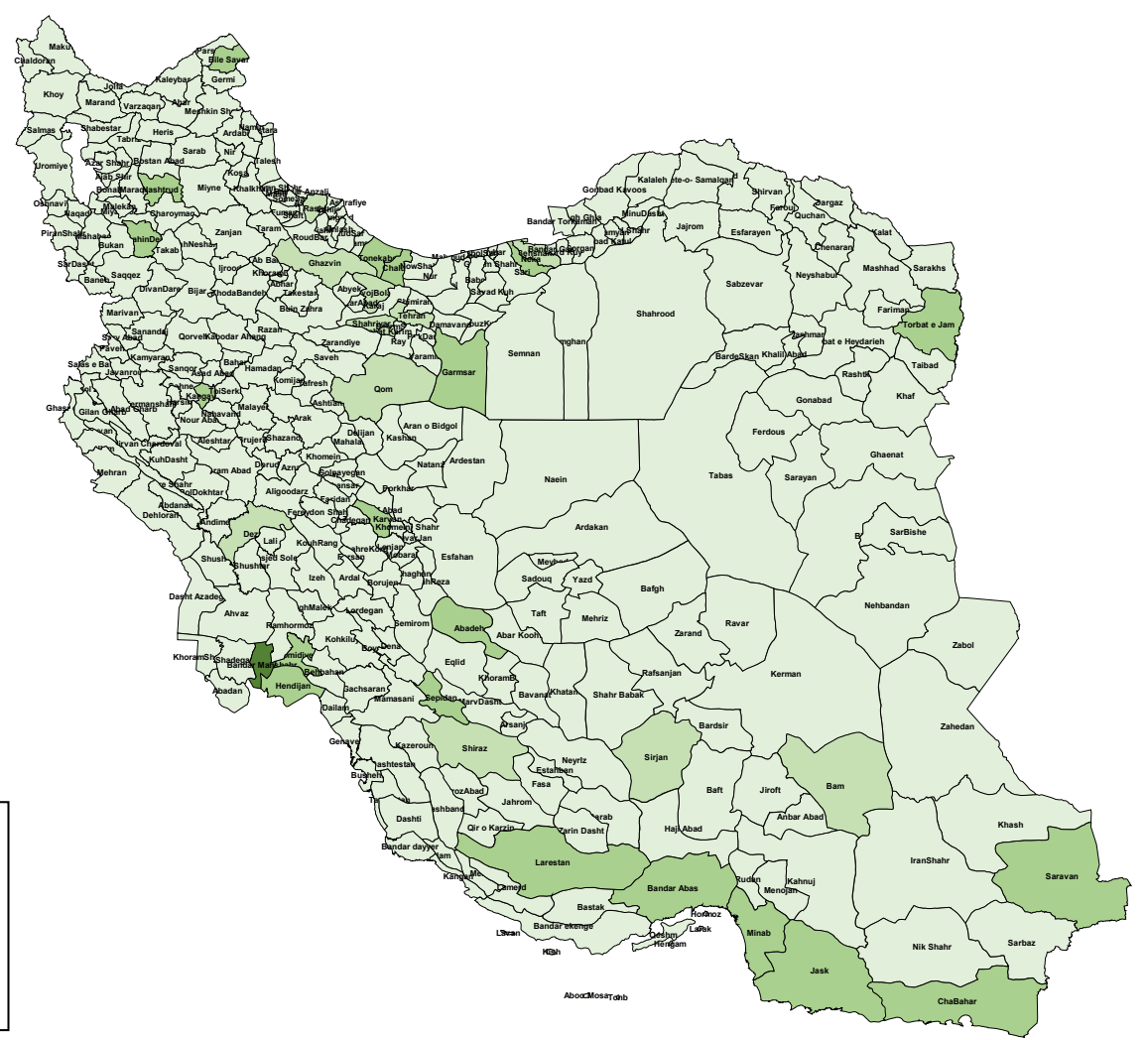

Fig. 2. The annual confirmed measles incidence per 1,000,000 in all Iranian districts area 


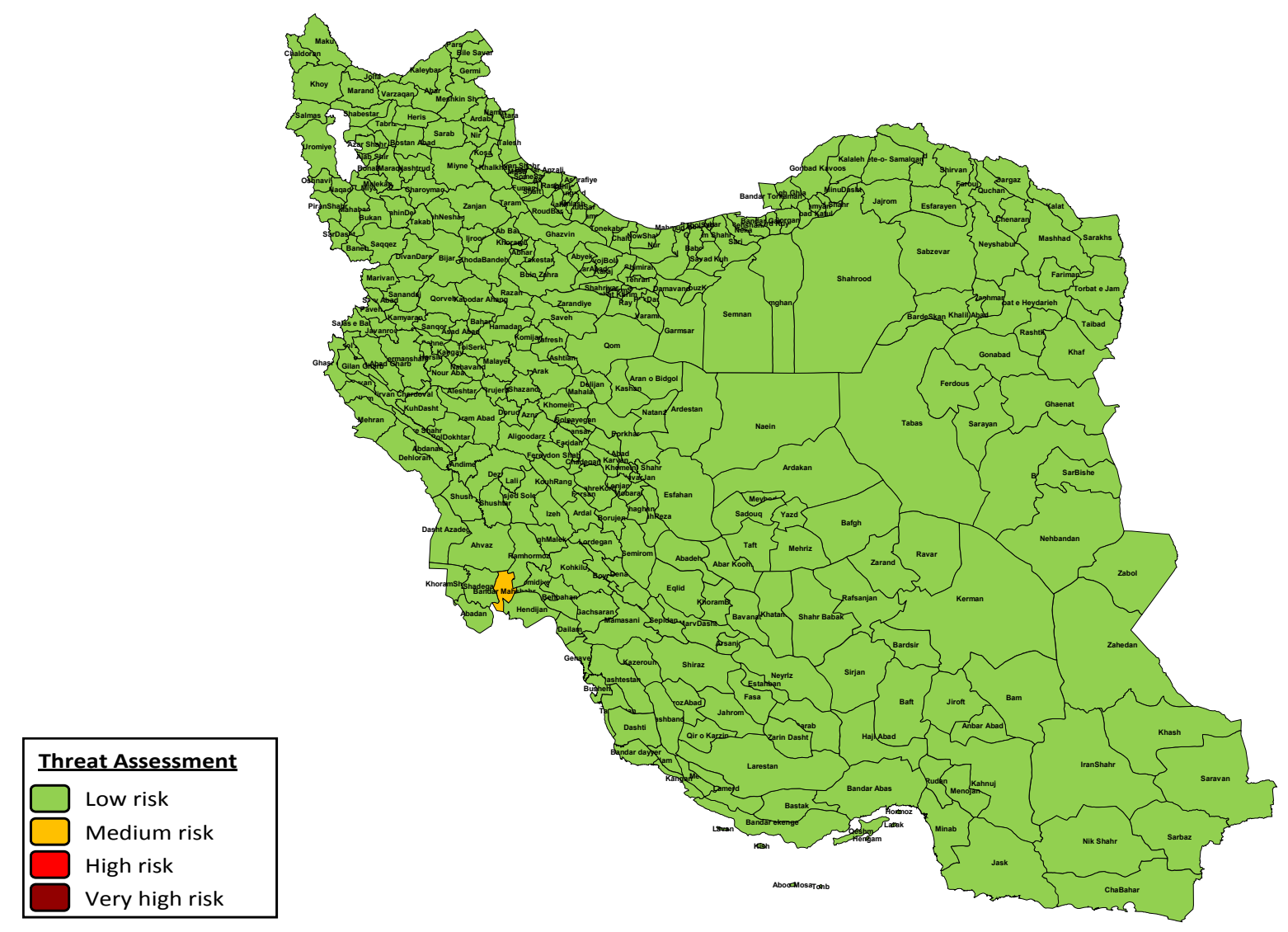

Fig. 3. Spatial mapping of Iranian districts based on threat assessment for measles outbreak

exept Bandar mahshahr, which was categorized as having a medum risk. Based on the opinion of the expert team in each district, in $36.6 \%$ of the districts, there was no vulnerable population. However, $30.8 \%$ of districts had $1 \mathrm{VPG}$, $22.5 \%$ had 2 VPGs, $8.6 \%$ had 3 VPGs, $0.9 \%$ had 4 VPGs, and $0.6 \%$ had 5 VPGs. Moreover, the districts with more than 3 risk points due to VPGs constitutued $10 \%$ of all districs of Iran. The spatial mapping of the districts based on threat assessment for measles outbreak is depicted in Figure 3.

\section{Discussion}

Results of this study showed that the annual incidence rate of measles in Iran was low during 2014 -2016 and the average annual incidence rate was 3.442 per 1 million population. The annaul incidence rate was 0.726 per 1 million population in 2014 and suddenly increased to more than 11-fold in 2015. Based on the evidence, the confirmed measles cases increased from 58 cases in 2014 to 668 cases in 2015. During 2015, a number of measles outbreaks occurred in different districts of Iran due to decreasing the immunity level and immigration, but by supplimentary immunization activities (SIAs), the immunization coverage increased and the measeles cases decreased to 90 cases in 2016 and the annual incidence rate decreased to 1.154 per 1 million population.

The measles elimination activities in Iran started after the catch-up campaign immunization of more than $50 \%$ of Iranians during 2003 and during a 5-year period after that campaign, from 2004 to 2008, the measles incidence decreased to 0.2 cases per 1 million and only 103 laboratory-confirmed measles cases were registered during that period (8). However, the confirmed measles cases increased each year after 2004 and reached to 668 cases in 2015 with some outbreaks. The outbreak of measles occured when there were more than 4 clinically suspected measles cases, or 2 laboratory confirmed cases were positive from 3 or more confirmed cases during 7 days (2, 20). Nevertheles, in Iran, each confirmed case of measles is defined as outbreak. However, implementation of complementary immunization activities in more than 45 districts in 8 medical universities, especially in deprived areas, reduced measles outbreak and annaual incidence rate in $2016(6,21)$.

The mean age of measles cases decreased during the study period from 9.48 years in 2014 to 9.32 years in 2015 and 6.9 years in 2016. Of the cases reported in 2014, only $25.9 \%$ were vaccinated and this rate increased to $51.1 \%$ in 2016. Also, athe results of this study showed that many of confirmed cases were unvaccinated or had unknown vaccine status in 2014 and the outbreaks occurred in 2015. According to recent studies in Iran, the immunization coverage of MMR1 and MMR2 is more than 95\% $(5,6)$; however, delay in MMR vaccinations in children may be due to some demographic charactristics $(9,11)$. However, routine immunization must be strengthened and the immunity gaps, especially in deprived and migrated areas should be identified and fully overed by SIAs $(6,22)$. In 
addition, according to the measles occurrence from 2004 to 2016, more attention should be paid to the WHO measles elimination goal (1).

According to the results of this study, the confirmed measles cases were reported in 33 districts of Iran in 2016 and based on the WHO measles risk assessment tool, all Iranian districts were depicted as low risk after threat assessment except Bandar-e- Mahshahr in Khuzestan province with highest annual confirmed measles incidence (92.9 per 1 million population). However, Sepidan in Fars province with 33 per 1 million population and Hedijan in Khuzestan with 25.8 per 1 million population were the second and third districts with highest annual confirmed measles incidence. Some studies have recently been conducted in developing countries using the WHO measles risk assessment tool $(1,16,17,23)$. Kriss et al study showed that in Romania, $64 \%$ of districts were categorized as very high or high risk for measles (16). Another study by Kriss et al revealed that $32 \%$ of health districts in Namibia are classified as high risk or very high risk (1). Ducusin et al study in Philippines showed that $48 \%$ of 122 districts and highly urbanized cities in the Philippines were classified as high risk or very high risk. Risk assessment results are helpful in monitoring the nationwide SIA, supervising the vaccination status (23), and guiding measles elimination strategies in areas that require improvement (1). Regular risk assessments of measles is helpful in planing the risk mitigation activities and measuring the effectiveness of measles elimination program (23) as well as monitoring the surveillance quality indictors (7). Annual assessments can provide valuable information for vaccination programs at the national level. Also, they can enhance measles elimination efforts in each district, including improving immunization services and vaccination campaigns and increasing surveillance $(7,16)$.

\section{Conclusion}

The average annual incidence rate of measles in Iran was low during 2014-2016; however, the measles incidence increased in 2015 due to some outbreaks that were controled after increasing the supplimentary vaccination coverage in high risk areas. Therefore, after a 11-fold increase in the number of measles cases in 2014, the number of measles cases decreased to more than 7-folds in 2016. However, maintaning a high immunization coverage for measles and increasing the percentage of people who received timely vaccination can be effective in reaching measles elimination goals.

\section{Conflict of Interests}

The authors declare that they have no competing interests.

\section{References}

1. Kriss JL, De Wee RJ, Lam E, Kaiser R, Shibeshi ME, Ndevaetela EE, et al. Development of the World Health Organization Measles Programmatic Risk Assessment Tool Using Experience from the 2009 Measles Outbreak in Namibia. Risk Anal. 2017;37(6):1072-81.

2. Zahraei SM, Gouya MM, Mokhtari Azad T, Soltanshahi R, Sabouri A, Naouri B, et al. Successful control and impending elimination of measles in the Islamic Republic of Iran. J Infect Dis. 2011;204(supp1_1):S305-S11.
3. Esteghamati A, Gouya MM, Zahraei SM, Dadras MN, Rashidi A, Mahoney F. Progress in measles and rubella elimination in Iran. Pediatr Infect Dis J. 2007;26(12):1137-41.

4. Karami M, Zahraei SM, Sabouri A, Soltanshahi R, Biderafsh A, Piri N, et al. Documentation of Measles Elimination in Iran: Evidences from 2012 to 2014. J Res Health Sci. 2017;17(3):e00387.

5.Zahraei SM, Eshrati B, Gouya MM, Mohammadbeigi A, Kamran A. Is there still an immunity gap in high-level national immunization coverage, Iran? Arch Iran Med. 2014;17(10):698-701.

6.Zahraei SM, Gouya MM, Mohammadi M, Tabatabaei SM, Zanganeh M, Zareban I, et al. A Survey on Measles and Rubella Supplementary Immunization Activities (SIAs) in Iran. Health Scope. 2017;6(4):e64184.

7.Zahraei SM, Mohammadbeigi A, Mohammadsalehi N, Sabouri A, Afrashteh S, Arsang Jang S, et al. Monitoring of Surveillance Quality Indicators of Measles in Iranian Districts: Analysis of Measles Surveillance System 2014-2016. J Res Health Sci. 2018;18(3):e00418.

8. Zahraei S, Gouya M, Mokhtari Azad T, Dadras M, Hodaei P, Sabouri A. Descriptive epidemiology of measles in Iran, 2004-2008. Iran J Infect Dis Trop Med 2009;14:1-5. [In Persian]

9. Rejali M, Mohammadbeigi A, Mokhtari M, Zahraei SM, Eshrati B. Timing and delay in children vaccination; evaluation of expanded program of immunization in outskirt of Iranian cities. J Res Health Sci. 2015;15(1):54-8.

10. Jadidi R, Mohammadbeigi A, Mohammadsalehi N, Ansari H, Ghaderi E. Inequity in timeliness of MMR vaccination in children living in the suburbs of Iranian cities. Int J Biomed Sci. 2015;11(2):93-8.

11. Mohammadbeigi A, Mokhtari M, Zahraei SM, Eshrati B, Rejali M. Survival analysis for predictive factors of delay vaccination in iranian children. Int J Prev Med. 2015;1(6):119.

12. Zahraei SM, Mohammadbeigi A, Mohammadsalehi N, Sabouri A, Afrashteh S, Arsang Jang S, et al. Monitoring of Surveillance Quality Indicators of Measles in Iranian Districts: Analysis of Measles Surveillance System 2014-2016. J Res Health Sci. 2018;18(3).

13. Statistical Centre of Iran. National Population and Housing Census, 2011: Selected Findings/Vice Presidency for Strategic Planning and Supervision. Tehran; 2011.

14. World Health Organization (WHO). Measles Risk Assessment Tool, Setup guide. 2017: URL: http://www.who.int/immunization/ monitoring_surveillance/routine/Measles_Risk_Assessment_Tool_set up guide V1.5 EN.pdf?ua=1.

15. World Health Organization. WHO-recommended surveillance standard of measles. 2010. p. URL: https://www.who.int/ immunization/monitoring_surveillance/burden/vpd/surveillance_type/ active/measles_standards/en/.

16. Kriss JL, Stanescu A, Pistol A, Butu C, Goodson JL. The World Health Organization Measles Programmatic Risk Assessment ToolRomania, 2015. Risk Anal. 2017;37(6):1096-107.

17. Lam E, Schluter WW, Masresha BG, Teleb N, Bravo-Alcantara P, Shefer A, et al. Development of a District-Level Programmatic Assessment Tool for Risk of Measles Virus Transmission. Risk Anal. 2017;37(6):1052-62.

18. World Health Organization (WHO). Measles Programmatic Risk Assessment Tool. 2017: URL: http://www.who.int/entity/ immunization/monitoring_surveillance/routine/Measles_Risk_Assess ment Tool v1.7.xlsm

19. Mohammadbeigi A, Zahraei SM, Asgarian A, Afrashteh S, Mohammadsalehi N, Khazaei S, et al. Estimation of measles risk using the World Health Organization Measles Programmatic Risk Assessment Tool, Iran. Heliyon. 2018;4(11):e00886.

20. World Health Organization. Measles elimination field guide: Manila: WHO Regional Office for the Western Pacific; 2013.

21. Izadi S, Zahraei SM, Mokhtari-Azad T. Seroprevalence of antibodies to measles and rubella eight months after a vaccination campaign in the southeast of Iran. Hum Vaccin Immunother. 2018;14(6):1412-6.

22. Ma C, Hao L, Zhang Y, Su Q, Rodewald L, An Z, et al. Monitoring progress towards the elimination of measles in China: an analysis of measles surveillance data. Bull World Health Organ. 2014;92(5):340-7.

23. Ducusin MJU, de Quiroz-Castro M, Roesel S, Garcia LC, Cecilio-Elfa D, Schluter WW, et al. Using the World Health Organization Measles Programmatic Risk Assessment Tool for Monitoring of Supplemental Immunization Activities in the Philippines. Risk Anal. 2017;37(6):1082-95. 